\title{
Nonlinear Free Vibrations of Coupled Spans of Overhead Transmission Lines
}

\author{
Sjoerd W. Rienstra \\ Department of Mathematics and Computing Science, \\ Eindhoven University of Technology, The Netherlands \\ s.w.rienstra@tue.nl
}

\begin{abstract}
The weakly nonlinear, freely vibrating motion of a system of coupled spans of suspended overhead transmission lines is studied. It is shown that the natural vibration is the gravity mode, of which the unsteady tension component vanishes in the first harmonic. The problem was born out of a study of the phenomenon of galloping, which is a high amplitude periodic oscillation of overhead transmission lines due to steady cross wind. Particular attention is given to an intermodal resonance, which may be interesting for galloping control.
\end{abstract}

\section{Introduction}

Overhead transmission lines for transport of high-voltage electricity are cables made of aluminium alloy, suspended between high towers in the countryside. One cable of several kilometers between two anchoring towers and carried by several towers in a row is called a section. The part of a section that is suspended between two towers is called a span. As the cable is connected to the towers by a freely movable suspension string or isolator, the dynamical motion of neighbouring spans are coupled. In wintertime when the cable is covered by snow or ice, the cables are vulnerable to large scale vertical vibrations in combination with a torsional vibration sustained by steady cross winds. This aero-elastic instability is known as galloping [1-5]. For high enough amplitudes neighbouring conductors may get close enough for the air-insulation to break down, causing a short-circuit and structural damage to the cables.

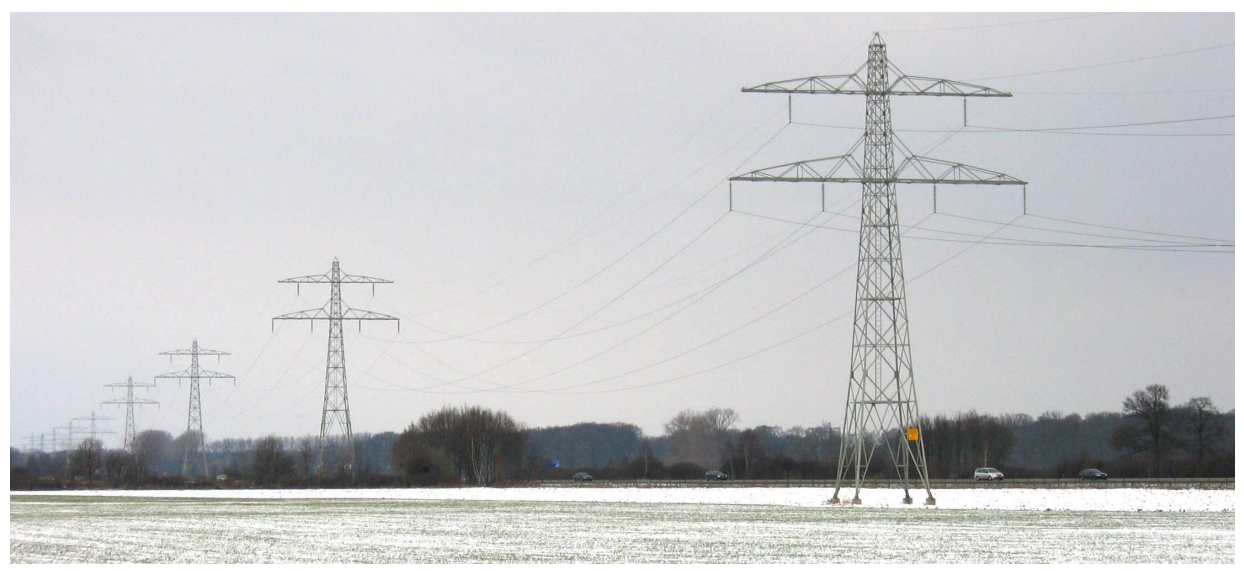

Figure 1: Overhead transmission lines. Spans are suspended between towers from suspension strings. 
It is known from observations that even a small wind force is sufficient to maintain a galloping vibration. The motion of the cable is therefore very close to a free vibration, which is what we will consider here, although a more complete modelling would include the driving force of the wind and the effect of friction with the air [6].

Both the torsional motion and the horizontal cable deflection are known to be important for the full problem, but we will concentrate on the asymptotic analysis of the coupling of spans. Therefore, torsion will be assumed to be decoupled from the vertical vibration and the horizontal motion to be negligible.

The paper is organised in four parts. First, we derive a systematic model by asymptotic reduction of a full model. This full model, based on first principles of Newton's and Hooke's law, assumes a linear elastic cable without bending stiffness moving in a vertical plane in a frictionless medium. The asymptotic reduction utilizes two small parameters, viz. geometrical slenderness (small sag/span ratio) and a high elastic stiffness (small span/ longitudinal wave length ratio). For a proper asymptotic analysis these two parameters have to be coupled. The resulting equations are similar, but not exactly the same, as what has been presented elsewhere like in $[7,8]$; in particular their asymptotic treatment of the $x$-component is inaccurate or at least unclear, which becomes important for higher orders. Our systematic derivation has been presented earlier in [3], but for clarity we will repeat the arguments here.

The second part consists of a Lindstedt-Poincaré approximation of weakly nonlinear transversal wave motion, where the small parameter is now the dimensionless amplitude. It is shown that the natural motion in a coupled series of spans is not of elasto-gravity type (the usually adopted galloping motion in a single span) but of gravity type. As most galloping occurs with series of spans, the relevance of single span motion is therefore questionable. A result, reported already in [3], is the presence of intermodal resonance for certain choices of parameters.

This resonance has been studied in the third part. It is shown that near this resonance no motion is possible consisting of a dominating gravity mode. The second harmonic of elasto-gravity type is just as big or bigger. It is conjectured that designing the suspended cable with parameter values right at this resonance will delay or hinder the occurrence of galloping.

In the fourth part some example cases are evaluated and illustrated by figures.

\section{Model}

\subsection{Differential equations and boundary conditions}

Consider a cable, fixed at the outer ends, and divided into $N$ equal spans by $N-1$ equal supports (see figure 2). $N$ is not large (in the asymptotic sense to be explained below). The supports are inextensible suspension strings (isolators) of length $a$ and negligible weight, suspended from fixed pivots separated by a distance $S$, the span size. 


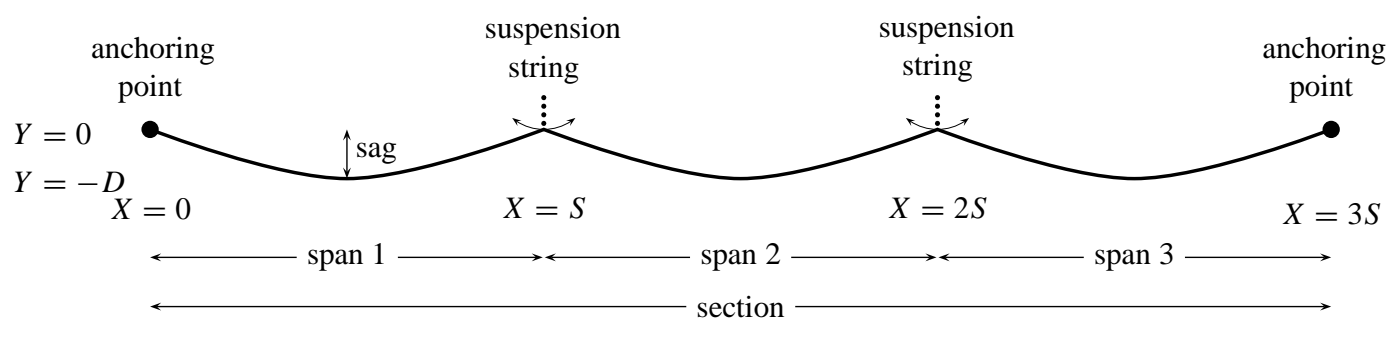

Figure 2: Sketch of a suspended cable, consisting of 3 interconnected spans.

The cable is linearly elastic, with negligible bending stiffness, of uniform undeformed effective crosssection $\mathcal{A}$, mass per unit length $m$, and Young's modulus $E$. It has a length per span $L$ when the cable is free of tension. Stationary, each span has a sag $D$ which depends on $S$ and $L$. In practice, the prescribed sag $D$ is obtained by applying a suitable cable tension, from which length $L$ follows. So $L$ is an unknown of the stationary problem. This, however, has no bearing at all on the unsteady problem.

We parametrize the position along the length of the cable by the variable $\ell \in[0, N L]$, such that this is just the arc length when the cable is unstretched. The (dimensional) time variable is $t$. We will here only consider cable motion in a vertical plane, which is provided with a Cartesian coordinate system oriented such that the gravity vector $-g \boldsymbol{e}_{y}$ points into the negative $y$-direction (in reality a point on the cable moves along a slightly tilted vertical ellipse).

The cable position is given by the position vector $X(\ell, t)=(X(\ell, t), Y(\ell, t))$ with a corresponding tension vector $\boldsymbol{T}(\ell, t)=T(\ell, t)(\cos \psi, \sin \psi)$, where $\psi$ is the positively oriented angle between the cable tangent and the horizontal. The tension vector is tangent to the cable because of the assumed negligible bending stiffness.

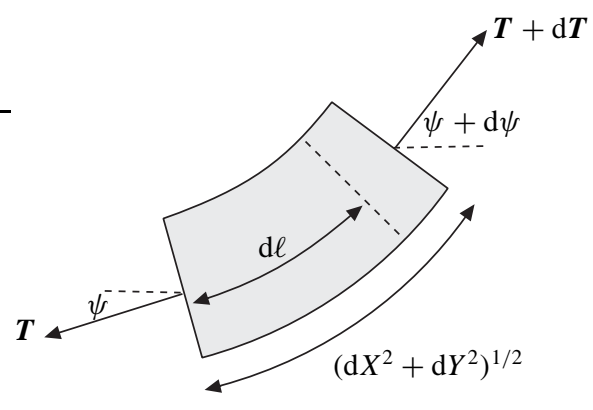

Figure 3: Sketch of cable element stretched under tension

Now consider a small cable element $\mathrm{d} \ell$. Due to gravity, cable tension, and inertial forces, this element is stretched (but the mass remains the same). According to Hooke's law [9], a cable element is elongated in proportion to the tension (Figure 3), so

$$
\left(\mathrm{d} X^{2}+\mathrm{d} Y^{2}\right)^{1 / 2}=\left(1+\frac{T}{E \mathcal{A}}\right) \mathrm{d} \ell .
$$

According to Newton's law, the internal (tension) and external (gravity) forces are in equilibrium with the inertial forces, so

$$
\mathrm{d} \boldsymbol{T}=\left(g \boldsymbol{e}_{y}+\ddot{\boldsymbol{X}}\right) m \mathrm{~d} \ell,
$$


where ij denotes a second derivative with respect to time. When we introduce

$$
\frac{\partial X}{\partial \ell}=\left(1+\frac{T}{E \mathcal{A}}\right) \cos \psi, \quad \frac{\partial Y}{\partial \ell}=\left(1+\frac{T}{E \mathcal{A}}\right) \sin \psi,
$$

the equations that finally result in the limit $\mathrm{d} \ell \rightarrow 0$ are given by [7]

$$
\begin{aligned}
\frac{\partial}{\partial \ell}\left(\frac{T}{1+T / E \mathcal{A}} \frac{\partial X}{\partial \ell}\right) & =m \frac{\partial^{2} X}{\partial t^{2}} \\
\frac{\partial}{\partial \ell}\left(\frac{T}{1+T / E \mathcal{A}} \frac{\partial Y}{\partial \ell}\right) & =m \frac{\partial^{2} Y}{\partial t^{2}}+m g, \\
\left(\frac{\partial X}{\partial \ell}\right)^{2}+\left(\frac{\partial Y}{\partial \ell}\right)^{2} & =\left(1+\frac{T}{E \mathcal{A}}\right)^{2} .
\end{aligned}
$$

The boundary and coupling conditions are (see Figure 4)

(i) fixed supports at $\ell=0$ and $\ell=N L$.

$$
\begin{array}{ll}
X=0, \quad Y=0 & (\ell=0), \\
X=N S, Y=0 \quad(\ell=N L),
\end{array}
$$

(ii) at the suspension strings $\ell=n L$, where $n=1, \ldots, N-1$, position vector $\boldsymbol{X}$ is continuous, the rigid but hinged suspension strings restrict the motion to a circle, while the force component normal to the string is continuous.

$$
\begin{gathered}
{[\boldsymbol{X}]_{\ell=n L-}^{\ell=n L+}=0,} \\
(X-n S)^{2}+(Y-a)^{2}=a^{2}, \\
{[T \cos (\phi-\psi)]_{\ell=n L-}^{\ell=n L+}=0 .}
\end{gathered}
$$

$\phi$ denotes the positively oriented angle of the suspension string with the vertical and $\ell=$ $n L \pm$ denote the limits from either side of the support.

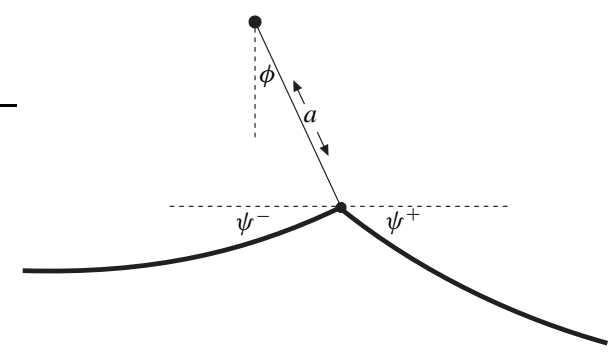

Figure 4: Sketch of cables connected at suspension string

\subsection{Small parameters and the mode of vibration}

The type of motion of interest allows further reduction of the model, and is specified by 
- The ratio of sag $D$ (the maximum stationary vertical displacement) and cable length $L$ is small (typically in the order of $\frac{1}{30}$ ), so the slenderness

$$
\varepsilon=D / L \rightarrow 0
$$

is a small parameter in the problem. This parameter will be used later to further reduce the problem.

- The total vertical non-stationary displacement is of the order of the sag, so

$$
Y / L=\mathcal{O}(\varepsilon) .
$$

- The transversal wave length $\lambda_{T}$ is of the order of $L$, so

$$
\lambda_{T} / L=\mathcal{O}(1) .
$$

This is exactly what may be expected from the boundary conditions at the span ends: $\frac{1}{2}, 1,2$, etc. waves per span.

- Apart from the slenderness, there is still another small parameter in the problem, which is less visible, as it is related to the relative elasticity.

In general, it takes some time before a change of tension has been distributed, as a longitudinal tensional wave, through the cable. This time, however, is very short, since the propagation speed of these waves (the sound speed) is high and $N$ is not large. In other words, the longitudinal wave length $\lambda_{L}$ is large compared to $L$. A second small parameter, however, is usually not very convenient in an asymptotic analysis, so we use the estimate, which is consistent with practice, that

$$
L / \lambda_{L}=\mathcal{O}(\varepsilon)
$$

- The string length $a$ is of the order of the sag, so

$$
a / L=\mathcal{O}(\varepsilon) .
$$

- The relative amplitude $\delta$ of the time dependent perturbation will be taken small but bigger than the orders of $\varepsilon$ neglected. We will assume a nearly harmonic vibration, with a single dominating frequency $\omega$, which is to be found. Since we are interested in the intrinsic nonlinear interaction between the harmonics, we will analyse the generated higher harmonics by a Lindstedt-Poincaré series in $\delta$ [10-12]. To keep the results clear, higher harmonics not generated by the first one will be excluded.

\section{Asymptotic analysis}

\subsection{Reduced problem}

The basic small parameter $\varepsilon$ will be used to reduce the above general problem to an asymptotic model. This model, assumed independent of $\varepsilon$, will subsequently be analysed asymptotically for small relative amplitude $\delta$.

Since the longitudinal wave speed is $c_{L}=(E \mathcal{A} / m)^{1 / 2}$, while $L / \lambda_{L}=\omega L / c_{L}=\mathcal{O}(\varepsilon)$, we can introduce the reference frequency $\omega_{\text {ref }}=\varepsilon(E \mathcal{A} / m)^{1 / 2} / L$. The dimensionless frequency and time variable are then given by

$$
\omega=\omega_{\text {ref }} \omega^{*}, \quad t=t^{*} / \omega_{\text {ref }}
$$


Since $\lambda_{T} / L=\mathcal{O}(1)$ the spatial coordinate $\ell$ obviously scales on $L$ (note that $L$ is an unknown of the problem). Since $Y / L=\mathcal{O}(\varepsilon), Y$ scales on $\varepsilon L$. The transversal wave velocity is $c_{T}=(T / m)^{1 / 2}$, so $\lambda_{T} / L=c_{T} / \omega L=\mathcal{O}(1)$, yielding that the tension scales on $T_{\text {ref }}=\varepsilon^{2} E \mathcal{A}$. Together we have for $\ell \in\{$ the $n$-th span $\}$

$$
\ell=(n-1+s) L, \quad Y(\ell, t)=\varepsilon L Y^{*}\left(s, t^{*} ; n\right), \quad T(\ell, t)=T_{\text {ref }} T^{*}\left(s, t^{*} ; n\right),
$$

where we introduced a local nondimensional parameter $s \in[0,1]$ to parametrize the position within a span. The suitable scaling for $x$ is more subtle. Of course, the slender geometry suggests immediately that $X \approx \ell$. However, we are only interested in the $x$-displacement which is very small. By substituting above estimates in equation (4c), it transpires that $\frac{\partial}{\partial \ell} X=1+\mathcal{O}\left(\varepsilon^{2}\right)$, and so we write for $X$ from the $n$-th span

$$
X(\ell, t)=(n-1) S+L s+\varepsilon^{2} L X^{*}\left(s, t^{*} ; n\right) .
$$

Finally, we investigate the rôle of gravity. If we substitute the present estimates into equation (4b), we find the term $m g L / E \mathcal{A} \varepsilon^{3}$ next to terms of $\mathcal{O}(1)$. So it has to be $\mathcal{O}(1)$ or smaller. Suppose it is smaller, then the stationary solution would be to leading order $Y \equiv 0$, so $D=0$, which is contradictory to our scaling assumptions. So the term is $\mathcal{O}(1)$ and we introduce

$$
\mu=\frac{m g L}{8 E \mathcal{A} \varepsilon^{3}}=\mathcal{O}(1)
$$

(where the factor 8 is included for notational convenience; c.f. [7]). While we omit the superscript asterisks from here on, we obtain, under the approximation for small $\varepsilon$, the reduced version of problem (4) as follows

$$
\begin{aligned}
\frac{\partial T}{\partial s} & =0, \\
\frac{\partial}{\partial s}\left(T \frac{\partial Y}{\partial s}\right) & =8 \mu+\frac{\partial^{2} Y}{\partial t^{2}}, \\
\frac{\partial X}{\partial s}+\frac{1}{2}\left(\frac{\partial Y}{\partial s}\right)^{2} & =T .
\end{aligned}
$$

In practice, the sag $D$ and span size $S$ are known, while cable length $L$ is to be determined. So $\varepsilon$ and $\mu$ are to be determined from the stationary solution. Moreover, all problem parameters are assumed to be the same for all spans and so the stationary solution is periodic in space. It is therefore useful to split up the solution in a stationary and non-stationary part

$$
\begin{aligned}
& X(s, t ; n)=X_{0}(s)+x(s, t ; n), \\
& Y(s, t ; n)=Y_{0}(s)+y(s, t ; n), \\
& T(s, t ; n)=T_{0}(s)+\tau(s, t ; n),
\end{aligned}
$$

with boundary conditions for the stationary part: $Y_{0}(0)=Y_{0}(1)=0$, and $X_{0}(0)=0$. As we scaled $Y$ on $\varepsilon L=D$ and, from symmetry, anticipate the location of maximum deflection halfway, we have of course $Y_{0}\left(\frac{1}{2}\right)=-1$. The condition for $X_{0}$ at $s=1$ is not really a boundary condition to the scaled problem, but implicitly determines the unknown length $L$ by the relation $S=L\left(1+\varepsilon^{2} S_{0}\right)$ and (up to the $\varepsilon$-approximation pursued so far) $X_{0}(1)=S_{0}$. The result is easily found to be a parabola, and given by

$$
\begin{aligned}
T_{0}(s) & =\mu, \\
Y_{0}(s) & =-4\left(s-s^{2}\right), \\
X_{0}(s) & =\mu s-\frac{4}{3}-\frac{32}{3}\left(s-\frac{1}{2}\right)^{3}, \\
S_{0} & =\mu-\frac{8}{3} .
\end{aligned}
$$


If we substitute (17) and (18) into (16) we obtain our fundamental non-stationary problem

$$
\begin{aligned}
\frac{\partial \tau}{\partial s} & =0, \\
(\mu+\tau) \frac{\partial^{2} y}{\partial s^{2}}+8 \tau & =\frac{\partial^{2} y}{\partial t^{2}}, \\
\frac{\partial x}{\partial s}+8\left(s-\frac{1}{2}\right) \frac{\partial y}{\partial s}+\frac{1}{2}\left(\frac{\partial y}{\partial s}\right)^{2} & =\tau .
\end{aligned}
$$

The boundary conditions at the rigid supports follow readily:

$$
x(0, t ; 1)=x(1, t ; N)=y(0, t ; 1)=y(1, t ; N)=0 .
$$

For the conditions at the suspension string $(s=0, n=2,3, \ldots, N$ and $s=1, n=1,2, \ldots, N-1)$ we observe that $a \phi / L=\mathcal{O}\left(\varepsilon^{2}\right)$ as it is of the order of the $x$-variation, so $\phi=\mathcal{O}(\varepsilon)$, and we obtain for small $\varepsilon$ the conditions

$$
y(0, t ; n)=y(1, t ; n)=0, \quad x(1, t ; n)=x(0, t ; n+1), \quad \tau(1, t ; n)=\tau(0, t ; n+1),
$$

with the important consequences that

$$
\sum_{n=1}^{N}[x(1, t ; n)-x(0, t ; n)]=0, \quad \tau=\text { constant in } s \text { and independent of } n .
$$

\subsection{Weakly harmonic motion}

The Lindstedt-Poincaré method [10-12] involves the assumption of a periodic solution with fundamental, as yet unknown, frequency $\omega$. The amplitude $\delta$ will be introduced as a small parameter, and based on this a solution will be constructed as a power series in $\delta$, where $\omega$ is also dependent on $\delta$.

In order to apply the condition of periodicity it is necessary to introduce the inherent time scale

$$
t^{\prime}=\omega t
$$

Now assume $y=\mathcal{O}(\delta)$ and expand

$$
y=\delta y_{1}+\delta^{2} y_{2}+\ldots
$$

and similarly for $x$ and $\tau$. We consider $y_{1}, y_{2}, \ldots$ normalized, for example as $y_{1}\left(\frac{1}{2}\right)=y_{2}\left(\frac{1}{2}\right)=\ldots=$ 1. We expand also $\omega$ as

$$
\omega=\omega_{0}+\delta^{2} \omega_{2}+\ldots
$$

where we used already the result that $\omega_{1}=0$. This could have been guessed from the intuitive argument that $\omega$ should not depend on the sign of the vibrational amplitude.

Introduce the notation \{\}$^{\prime}=\frac{\partial}{\partial s}\{\}$, and \{\}$=\frac{\partial}{\partial t^{\prime}}\{\}$. Substitute the $\delta$-series into (19), and collect like powers of $\delta$ :

$$
\left.\begin{array}{rl}
\mu y_{1}^{\prime \prime}+8 \tau_{1} & =\omega_{0}^{2} \ddot{y}_{1}, \\
x_{1}^{\prime}+8\left(s-\frac{1}{2}\right) y_{1}^{\prime} & =\tau_{1}, \\
\mu y_{2}^{\prime \prime}+8 \tau_{2}+\tau_{1} y_{1}^{\prime \prime} & =\omega_{0}^{2} \ddot{y}_{2}, \\
x_{2}^{\prime}+8\left(s-\frac{1}{2}\right) y_{2}^{\prime}+\frac{1}{2}\left(y_{1}^{\prime}\right)^{2} & =\tau_{2},
\end{array}\right\}
$$


with $\tau_{1}, \tau_{2}, \ldots$ constant in $s$. The interesting solutions here are based on the harmonic solution of the linearized problem (i.e. for $y_{1}, x_{1}, \tau_{1}$ ). Since any phase shift in time is unimportant, we may assume $y_{1}, x_{1}, \tau_{1}$ to vary in time like $\sin t^{\prime}$. The next question is now what time dependence this induces in the second order. In equations (27) the terms $\tau_{1} y_{1}^{\prime \prime}$ and $\left(y_{1}^{\prime}\right)^{2}$ act as an inhomogeneous (source) term. Since they vary in time like $\left(\sin t^{\prime}\right)^{2}=\frac{1}{2}-\frac{1}{2} \cos 2 t^{\prime}$, we expect therefore a constant and a $\cos 2 t^{\prime}$ contribution. So we put

$$
\begin{aligned}
& y_{1}\left(s, t^{\prime} ; n\right)=y_{11}(s ; n) \sin t^{\prime}, \\
& y_{2}\left(s, t^{\prime} ; n\right)=y_{20}(s ; n)+y_{22}(s ; n) \cos \left(2 t^{\prime}\right),
\end{aligned}
$$

and similarly for $x$ and $\tau$.

By substituting (28) into (26) and (27) and collecting the harmonics yield

$$
\left.\begin{array}{rl}
\mu y_{11}^{\prime \prime}+8 \tau_{11}+\omega_{0}^{2} y_{11} & =0, \\
x_{11}^{\prime}+8\left(s-\frac{1}{2}\right) y_{11}^{\prime} & =\tau_{11},
\end{array}\right\}
$$

The boundary conditions are the same as for $x, y$ and $\tau$ (20), (21) and (22), but now taken for each harmonic independently.

\section{Solutions}

In spite of the many assumptions and reductions made, the possible solutions are still numerous. This, on the other hand, is exactly in agreement with reality, where the cables are known to possibly vibrate in many modes. This indeterminacy is also a very important problem to be dealt with in any numerical simulation.

For a single span, two important classes of motion can be distinguished: elasto-gravity waves and gravity waves [3]. In the first type the tension perturbations are to leading order non-zero and elasticity and gravity act both as restoring force. In the second type elasticity is only important in the higher orders, and to leading order the tension vanishes. The probably best studied cable motion is the symmetric mode with fixed ends (a single span mode) which is an example of an elasto-gravity mode [3, 5, 7, 8, 13-17]. Higher order, asymmetric modes of singles spans may be of gravity-mode type.

For multiple spans the situation is actually simpler, because elasto-gravity waves are very unlikely to exist in practice. This is seen as follows. For $\tau_{11} \neq 0$, the solution of (29), at the $n$-th span, is given by

$$
\begin{aligned}
& y_{11}=\frac{16 \tau_{11}}{\omega_{0}^{2} \cos \left(\frac{1}{2} k\right)} \sin \left(\frac{1}{2} k s\right) \sin \left(\frac{1}{2} k-\frac{1}{2} k s\right), \\
& x_{11}=B_{n}+\frac{64 \tau_{11}}{\omega_{0}^{2} \cos \left(\frac{1}{2} k\right)}\left(\frac{\sin \left(k s-\frac{1}{2} k\right)}{k}-\left(s-\frac{1}{2}\right) \cos \left(k s-\frac{1}{2} k\right)\right),
\end{aligned}
$$


where modal wave number $k=\omega_{0} / \mu^{1 / 2}$ and the constant $\tau_{11}$ determines the amplitude of the mode. Condition (22) requires that $k$ is equal to any solution of

$$
\frac{64 N \tau_{11}}{\omega_{0}^{2}}\left(\frac{\tan \frac{1}{2} k}{\frac{1}{2} k}-1\right)=0,
$$

while with continuity conditions (21) it follows that $B_{n}=0$.

We see that the only solution possible is a repetition of $N$ the same single-span solutions of elasto-gravity type. This is in general unlikely, given the fact that the cable motion is produced by an uncontrolled cause of natural origin, viz. the wind. So we conclude that for overhead transmission lines the natural motion for multi-span configurations is a gravity wave with $\tau_{11} \equiv 0$, which we will further consider here. It is a relatively little studied type of motion, although it contains an interesting phenomenon of intermodal resonance.

\subsection{Multi-span gravity waves}

As we indicated above, this class of solutions has a vanishing tension's first harmonic $\tau_{11}$. We have then for the $n$-th span $(1 \leq n \leq N)$ the general solution for the $j$-th mode

$$
\begin{aligned}
\tau_{11}= & 0, \\
y_{11}= & A_{n} \sin k s, \quad \text { where } k=\omega_{0} / \mu^{1 / 2} \text { and } k=k_{j}=(2 j-1) \pi, \\
x_{11}= & B_{n}-8 A_{n}\left(\frac{\cos k s}{k}+\left(s-\frac{1}{2}\right) \sin k s\right), \\
& \text { where } B_{1}=8 k^{-1} A_{1}, B_{n}=8 k^{-1}\left(2 A_{1}+2 A_{2}+\ldots+2 A_{n-1}+A_{n}\right), \\
\tau_{20}= & \frac{3}{16} \omega_{0}^{2} \overline{A^{2}} \\
y_{20}= & 4 \tau_{20} \mu^{-1}\left(s-s^{2}\right), \\
x_{20}= & C_{n}+\tau_{20} s+\frac{8}{3} \tau_{20} \mu^{-1}\left(1+8\left(s-\frac{1}{2}\right)^{3}\right)-\frac{1}{8} k^{2} A_{n}^{2}\left(s+\frac{\sin 2 k s}{2 k}\right), \\
& \text { where } C_{1}=0, C_{n}=\frac{1}{8} k^{2}(n-1) \overline{A^{2}}-\frac{1}{8} k^{2}\left(A_{1}^{2}+\ldots+A_{n-1}^{2}\right), \\
& \frac{1}{8} k^{2} \omega_{0}^{2} \overline{A^{2}} \\
& 16-\omega_{0}^{2}, \\
\tau_{22}= & -2 \tau_{22} \omega_{0}^{-2}(1-\cos 2 k s), \\
x_{22}= & D_{n}+\tau_{22} s-16 \tau_{22} \omega_{0}^{-2}\left(\frac{1}{2}+\left(s-\frac{1}{2}\right) \cos 2 k s-\frac{\sin 2 k s}{2 k}\right) \\
& \text { where } D_{1}=0, D_{n}=\frac{1}{8} k^{2}\left(A_{1}^{2}+\ldots+A_{n-1}^{2}\left(s+\frac{\sin 2 k s}{2 k}\right),\right. \\
&
\end{aligned}
$$

We used the notation

$$
\overline{A^{2}}=\frac{1}{N} \sum_{n=1}^{N} A_{n}^{2} .
$$


Note that $\delta A_{n}$, rather than $A_{n}$, is the modal amplitude, so we may normalise the amplitudes, for example by assuming

$$
\overline{A^{2}}=1 \text {. }
$$

From (22) it follows that the amplitudes are further restricted by the condition that

$$
\bar{A}=\frac{1}{N} \sum_{n=1}^{N} A_{n}=0 .
$$

For example, a 2-span configuration $(N=2)$ with equal vibrational energy in each span will have $A_{1}=-A_{2}=1$ under normalisation (38).

The frequency is to leading order equal to $\omega_{0}=(2 j-1) \pi \mu^{1 / 2}$. The higher order shift $\omega_{2}$ is to be determined by higher order equations.

\subsection{Intermodal resonance near $\omega=4$}

A striking property of the obtained solution is the singularity at $\omega_{0}=4$ in $\tau_{22}$, where the present approximation breaks down. Apparently, a near-harmonic $j$-th mode solution which is tension free in the first harmonic is not possible for any value of $\mu$ near $(4 /(2 j-1) \pi)^{2}$. Due to intermodal resonance, the second mode of the second harmonic is amplified to first order whenever $\omega_{0}=4+\mathcal{O}(\delta)$.

In order to investigate the behaviour of the solution near this singularity we write $\mu=\mu_{0}+\delta \mu_{1}$, where $\mu_{0}=(4 / \pi)^{2}$ and assume $\omega=\omega_{0}+\delta \omega_{1}+\ldots$ (due to the asymmetric dependence on $\delta$ through $\mu, \omega_{1}$ is not necessarily zero here), to get

$$
\left.\begin{array}{rl}
\mu_{0} y_{1}^{\prime \prime}+8 \tau_{1} & =\omega_{0}^{2} \ddot{y}_{1}, \\
x_{1}^{\prime}+8\left(s-\frac{1}{2}\right) y_{1}^{\prime} & =\tau_{1}, \\
\mu_{0} y_{2}^{\prime \prime}+8 \tau_{2}+\left(\mu_{1}+\tau_{1}\right) y_{1}^{\prime \prime} & =\omega_{0}^{2} \ddot{y}_{2}+2 \omega_{0} \omega_{1} \ddot{y}_{1}, \\
x_{2}^{\prime}+8\left(s-\frac{1}{2}\right) y_{2}^{\prime}+\frac{1}{2}\left(y_{1}^{\prime}\right)^{2} & =\tau_{2} .
\end{array}\right\}
$$

In view of the above results we expect that a $\cos 2 t^{\prime}$-term is lifted from $\mathcal{O}\left(\delta^{2}\right)$-level to $\mathcal{O}(\delta)$-level, so we assume

$$
y_{1}\left(s, t^{\prime}\right)=y_{11} \sin t^{\prime}+y_{12} \cos 2 t^{\prime},
$$

and similarly for $x_{1}$ and $\tau_{1}$. After substitution and collecting the harmonics we find

$$
\left.\begin{array}{rl}
\mu_{0} y_{11}^{\prime \prime}+8 \tau_{11}+\omega_{0}^{2} y_{11} & =0, \\
x_{11}^{\prime}+8\left(s-\frac{1}{2}\right) y_{11}^{\prime} & =\tau_{11},
\end{array}\right\}
$$

Again, the boundary conditions (20), (21) and (22) are the same as for $x, y$ and $\tau$ but now taken for each harmonic independently. We are in particular interested in the problem with $\tau_{11} \equiv 0$. As we saw before, this yields the solution

$$
y_{11}=A_{n} \sin \pi s
$$




$$
\begin{aligned}
\tau_{11} & =0, \\
y_{12} & =-\frac{1}{8} \tau_{12}(1-\cos 2 \pi s),
\end{aligned}
$$

where $k=\pi, \omega_{0}=\mu_{0}^{1 / 2} k=4$, and $\bar{A}=0$ and an irrelevant multiple of $\sin 2 \pi s$ in $y_{12}$ is ignored. Tension $\tau_{12}$ will be determined from the higher order equations below. The coupling term $\left(y_{1}^{\prime}\right)^{2}$ generates $\sin t^{\prime}, \sin 3 t^{\prime}, \cos 2 t^{\prime}, \cos 4 t^{\prime}$ and constant terms, so we write for the next order

$$
y_{2}\left(s, t^{\prime}\right)=y_{20}+y_{21} \sin t^{\prime}+y_{22} \cos 2 t^{\prime}+y_{23} \sin 3 t^{\prime}+y_{24} \cos 4 t^{\prime},
$$

and similarly for $x$ and $\tau$. We obtain the systems of equations

$$
\left.\begin{array}{rl}
\mu_{0} y_{20}^{\prime \prime}+8 \tau_{20} & =0, \\
x_{20}^{\prime}+8\left(s-\frac{1}{2}\right) y_{20}^{\prime}+\frac{1}{4}\left(y_{11}^{\prime}\right)^{2}+\frac{1}{4}\left(y_{12}^{\prime}\right)^{2}=\tau_{20}, & \\
\mu_{0} y_{21}^{\prime \prime}+8 \tau_{21}+\mu_{1} y_{11}^{\prime \prime}+\omega_{0}^{2} y_{21}+2 \omega_{0} \omega_{1} y_{11} & =0, \\
x_{21}^{\prime}+8\left(s-\frac{1}{2}\right) y_{21}^{\prime}-\frac{1}{2} y_{11}^{\prime} y_{12}^{\prime} & =\tau_{21},
\end{array}\right\}
$$

This has the following solutions (for the $n$-th span).

$$
\begin{aligned}
y_{20} & =\frac{1}{4} \pi^{2} \tau_{20}\left(s-s^{2}\right), \\
\tau_{20} & =\frac{\frac{1}{128} \pi^{2}\left(\tau_{12}^{2}+16 \overline{A^{2}}\right)}{1+\frac{1}{3} \pi^{2}}, \\
y_{21} & =0 \\
\tau_{21} & =0 \\
\omega_{1} & =\frac{1}{8} \pi^{2} \mu_{1}, \\
y_{22} & =\frac{1}{8}\left(\tau_{22}-\frac{1}{16} \pi^{2} \mu_{1} \tau_{12}\right)(\cos 2 \pi s-1), \\
\tau_{12} & =-2 \overline{A^{2}} \mu_{1}^{-1}, \\
y_{23} & =0 \\
\tau_{23} & =0 \\
y_{24} & =\frac{1}{32} \tau_{24}(\cos 4 \pi s-1), \\
\tau_{24} & =\frac{1}{96} \pi^{2} \tau_{12}^{2},
\end{aligned}
$$

while $\tau_{22}$ is to be determined from the next order equations. We ignored the undriven multiples of $\sin \pi s, \sin 2 \pi s, \sin 3 \pi s$ and $\sin 4 \pi s$ in respectively $y_{21}, y_{22}, y_{23}$ and $y_{24}$. See the Appendix for the corresponding expressions for displacement $x$. 
The important conclusion is that the solution is, again, singular at $\mu_{1}=0$ through the tension $\tau_{12} \sim \mu_{1}^{-1}$. It follows that a solution, tension free in the first harmonic $\left(\tau_{1} \equiv 0\right)$, does not exist for $\mu=\mu_{0}+\mathcal{O}(\delta)$, while a solution which is tension free in the first mode of the first harmonic $\left(\tau_{11} \equiv 0\right)$ does not exist for $\mu=\mu_{0}+\mathcal{O}\left(\delta^{2}\right)$.

A speculative, but tempting conjecture is that a cable design with $\omega=4$ is favourable against galloping. The vibration energy of a galloping cable is probably highest at or near $\omega=4$ (with $y$ amplitudes kept the same), because it then includes a strong elastic component. As a result, it will be more difficult to initiate or maintain the process of galloping.

\section{Typical examples}

Two practically relevant examples of an oscillating, coupled, span are given in Figure 5. In both cases we have $m=1 \mathrm{~kg} / \mathrm{m}, g=9.8 \mathrm{~m} / \mathrm{s}^{2}, E \mathcal{A}=2.156 \times 10^{7} \mathrm{~N}$, and $S=320 \mathrm{~m}$, while $A_{n}=\overline{A^{2}}=1$ and the lowest mode $(j=1)$ is selected.
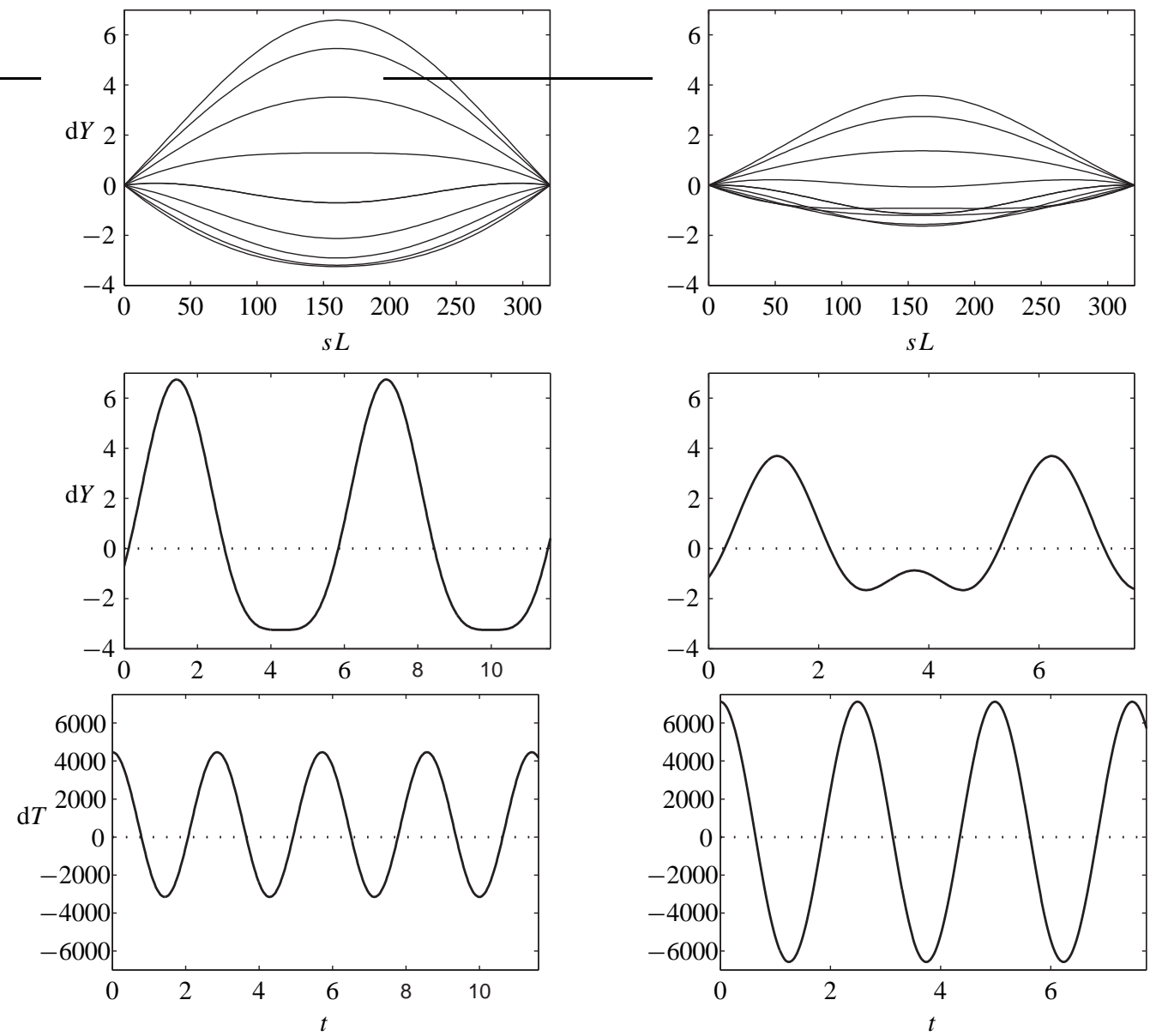

Figure 5: An oscillating cable span (coupled). From top to bottom: the vertical displacement $\mathrm{d} Y$ in $s L$ for various time steps; the position at the middle $\left(s=\frac{1}{2}\right)$ of the displacement in time; the varying part of the tension $\mathrm{d} T$ in time. All variables are dimensional.

Shown are the dimensional varying parts of vertical displacement $\mathrm{d} Y=\varepsilon L y$ and tension $\mathrm{d} T=$ 
$\varepsilon^{2} E \mathcal{A} \tau$. In the left column we have a stationary tension $T=12600 \mathrm{~N}$ and sag $D=10 \mathrm{~m}$ with $\delta=0.5$. In this case $L=320.7 \mathrm{~m}, \mu=0.6011, \varepsilon=0.0312$ and a frequency of $\omega=2.436$ or $0.175 \mathrm{~Hz}$. We observe a mild contribution of the higher harmonics in $\mathrm{d} Y$. These are more present in the case displayed in the right column where we have a $T=16500 \mathrm{~N}$ and $D=7.61 \mathrm{~m}$ with $\delta=0.3$. Now $L=320.3 \mathrm{~m}, \mu=1.354, \varepsilon=0.0238$ and a frequency of 3.655 or $0.201 \mathrm{~Hz}$.

Note that we have not included a near-resonance solution because tension component $\tau_{22}$ is still to be determined.

\section{Conclusions}

The problem of oscillating overhead transmission lines is considered in a strictly asymptotic way. The model, initially derived from first principles, is reduced by systematically exploiting two small parameters, viz. geometrical slenderness and relative elasticity, which allow us to remove irrelevant elements. In the final model all parts contribute significantly. Then an asymptotic solution of this model is obtained based on the assumption that the cable vibrates harmonically with weakly nonlinear perturbations.

The problem of interest here is a multi-span configuration with gravity waves, which is shown to be the natural wave form for coupled spans. For certain values of the problem parameter, say $\mu=\mu_{0}$, this solution appears to show intermodal resonance, where the solution breaks down. Some more detailed analysis showed that a solution, tension free in the first harmonic, does not exist for $\mu=\mu_{0}+\mathcal{O}(\delta)$, while a solution which is tension free in the first mode of the first harmonic does not exist for $\mu=\mu_{0}+\mathcal{O}\left(\delta^{2}\right)$.

The non-existence of a simple, harmonic wave form at this choice of parameters may be advantageous when galloping is to be avoided.

\section{Appendix}

For completeness, we give here the solutions of the $x$-displacements corresponding to the nearresonance problem of section 4.2 .

$$
\begin{aligned}
& x_{11}=B_{n}-8 A_{n}\left(\frac{\cos \pi s}{\pi}+\left(s-\frac{1}{2}\right) \sin \pi s\right) \\
& x_{12}=\tau 12\left(\frac{\sin 2 \pi s}{2 \pi}+\left(s-\frac{1}{2}\right)(1-\cos 2 \pi s)\right), \\
& x_{20}=C_{n}+\tau_{20} s \pi^{2}\left(\frac{4}{3} s^{2}-2 s+1\right)+\tau_{20} s-\frac{1}{8} \pi^{2} A_{n}^{2}\left(\frac{\sin 2 \pi s}{2 \pi}+s\right)+\frac{1}{128} \pi^{2} \tau_{12}^{2}\left(\frac{\sin 4 \pi s}{4 \pi}-s\right) \\
& x_{21}=D_{n}+\frac{1}{16} \pi A_{n} \tau_{12}\left(\cos \pi s+\frac{1}{3} \cos 3 \pi s\right), \\
& x_{22}=E_{n}+\tau_{22} s+\left(\tau_{22}-\frac{1}{16} \pi^{2} \mu_{1} \tau_{12}\right)\left(\frac{\sin 2 \pi s}{2 \pi}-\left(s-\frac{1}{2}\right) \cos 2 \pi s\right)+\frac{1}{8} \pi^{2} A_{n}^{2}\left(\frac{\sin 2 \pi s}{2 \pi}+s\right), \\
& x_{23}=F_{n}-\frac{1}{16} \pi A_{n} \tau_{12}\left(\cos \pi s+\frac{1}{3} \cos 3 \pi s\right), \\
& x_{24}=G_{n}+\tau_{24} s+\frac{1}{4} \tau_{24}\left(\frac{\sin 4 \pi s}{4 \pi}-\left(s-\frac{1}{2}\right) \cos 4 \pi s\right)+\frac{1}{128} \pi^{2} \tau_{12}^{2}\left(\frac{\sin 4 \pi s}{4 \pi}-s\right) .
\end{aligned}
$$

The integration constants $B_{n}, \ldots, G_{n}$ (not the same as in (34-36)) follow readily from the boundary and continuity conditions at $s=0$ and $s=1$. 


\section{References}

[1] J.P. den Hartog, Transmission line vibration due to sleet, Transactions of the American Institute of Electrical Engineers, 51, p.1074-1077, 1932

[2] A. Simpson, Wind-induced Vibration of Overhead Power Transmission Lines, Sci. Prog. Oxford 68, p.285-308, 1983.

[3] S.W. Rienstra, Non-Linear Free Vibrations Of Coupled Spans Of Suspended Cables, Proceedings of the Third European Conference on Mathematics in Industry, August 27-31 1988 Glasgow, Edited by J. Manley et al., 1990, Kluwer Academic Publishers and B.G. Teubner Stuttgart, pp.133-144

[4] O. Chabart, J.L. Lilien, Galloping of Electrical Lines in Wind Tunnel Facilities, Journal of Wind Engineering and Industrial Aerodynamics 74-76. p.967-976, 1998.

[5] C.B. Rawlins, Effect of non-linearity in free large oscillations of a shallow catenary, Journal of Sound and Vibration 273, p.857-874, 2004.

[6] A.H.P. van der Burgh, An Asymptotic Theory for the Free Vibrations of an Iced Two-Conductor Bundled Transmission Line, Asymptotic Analysis II - Surveys and Trends, p.413-430, Edited by F. Verhulst, Lecture Notes in Mathematics 985, Springer-Verlag, Berlin 1983

[7] H.M. Irvine, T.K. Caughey, The Linear Theory of Free Vibrations of a Suspended Cable, Proceedings of the Royal Society of London A341, p.299-315, 1974

[8] P. Hagedorn, B. Schäfer, On Non-linear Free Vibrations of an Elastic Cable, International Journal of Non-linear Mechanics 15, p.333-340, 1980

[9] L.D. Landau, E.M. Lifshitz, Theory of Elasticity, 2nd edition, Pergamon Press, Oxford, 1970.

[10] A.H. Nayfeh, Perturbation Methods, John Wiley \& Sons, Inc., New York, 1973

[11] J. Kevorkian, J.D. Cole, Multiple Scale and Singular Perturbation Methods, Springer, New York, 1996.

[12] A.H. Nayfeh, D.T. Mook, Nonlinear Oscillations, John Wiley \& Sons, New York, 1979

[13] A. Simpson, On the Oscillatory Motions of Translating Elastic Cables, Journal of Sound and Vibration 20, p.177-189, 1972.

[14] A. Luongo, G. Rega, F. Vestroni, Planar Non-Linear Vibrations of an Elastic Cable, International Journal of Non-linear Mechanics 19, p.39-52, 1984

[15] G. Rega, F. Vestroni, F. Benedettini, Parametric Analysis of Large Amplitude Free Vibrations of a Suspended Cable, International Journal of Solids and Structures 20, p.95-105, 1984.

[16] F. Benedettini and G. Rega, Non-linear Dynamics of an Elastic Cable under Planar Excitation, International Journal of Non-Linear Mechanics, 22(6), p.497-509, 1987.

[17] J.J. Burgess and M.S. Triantafyllou, The Elastic Frequencies of Cables, Journal of Sound and Vibration 120, p.153-165, 1988. 